\title{
Efeito agudo de diferentes estratégias de aquecimento sobre o desempenho do benchmark Fran em praticantes de Crossfit
}

\author{
Acute effects of different heating strategies on benchmark Fran performance in Crossfit
}

practitioners

Efecto agudo de diferentes estrategias de calentamiento sobre el rendimiento de benchmark Fran en practicantes de Crossfit

Recebido: 08/07/2021 | Revisado: 17/07/2021 | Aceito: 19/07/2021 | Publicado: 26/07/2021

\author{
Ana Keroly Rocha Freitas \\ ORCID: https://orcid.org/0000-0002-8721-7071 \\ Instituição Federal de Educação, Ciência e Tecnologia do Ceará, Brasil \\ E-mail: keroly.rocha.freitas@gmail.com \\ Nilson Vieira Pinto \\ ORCID: https://orcid.org/0000-0001-6548-8586 \\ Instituição Federal de Educação, Ciência e Tecnologia do Ceará, Brasil \\ E-mail: nilsonvieira@ifce.edu.br
}

\begin{abstract}
Resumo
O aquecimento é uma importante estratégia preparatória para o bom desempenho físico durante o treinamento, todavia a sua aplicação metodológica no CrossFit ainda é pouco discutida. Este estudo objetivou verificar o efeito agudo de diferentes estratégias de aquecimento sobre o desempenho do benchmark Fran em praticantes de CrossFit. Trata-se de um estudo com delineamento transversal e com abordagem quantitativa, composto por uma amostragem nãoprobabilística intencional de praticantes de CrossFit. Inicialmente foram avaliados o peso, altura, o índice de massa corporal e o percentual de gordura para fins de caracterização amostral. Em sequência foi realizado o teste de 1-RM para determinação da carga e intensidade de treinamento individual. Após obtenção de dados diagnósticos, foram aplicados, em dias distintos, o alongamento estático ativo (AL) e o aquecimento específico (AQ) seguido da avaliação de desempenho do benchmark "Fran", através da avaliação da frequência cardíaca (FC), pressão arterial sistólica (PAS) e diastólica (PAD) e escala de Borg. Os resultados caracterizaram uma amostragem com parâmetros antropométricos e de composição corporal homogênea. $\mathrm{O}$ aquecimento específico apresentou uma menor resposta da frequência cardíaca $(168,65 \pm 16,9 \mathrm{bpm})$ e da percepção subjetiva de esforço $(6,65 \pm 1,26)$ em relação ao alongamento estático $(178,8 \pm 13,50$ e $7,75 \pm 1,11$, respectivamente), entretanto as respostas pressóricas não foram estatisticamente diferentes entre si (PAS: $151,5 \pm 27,39 \mathrm{mmHg}$ vs. $154,5 \pm 10,99 \mathrm{mmHg}$; PAD: $116 \pm 5,98 \mathrm{mmHg}$ vs. $117,5 \pm 10,69$ mmHg. Em conclusão, o aquecimento específico promoveu um menor trabalho cardiovascular após a execução do benchmark em comparação ao alongamento estático.
\end{abstract}

Palavras-chave: Treinamento intervalado de alta intensidade; Exercício de aquecimento; Exercícios de alongamento muscular.

\begin{abstract}
Warming up is an important preparatory strategy for good physical performance during training, however its methodological application in CrossFit is still little discussed. This study aims to verify the acute effect of different warm-up strategies on benchmark Fran performance in CrossFit practitioners. This is a cross-sectional study with a quantitative approach, composed of an intentional non-probabilistic sampling of CrossFit practitioners. Initially, weight, height, body mass index and fat percentage will be evaluated for sample characterization purposes. Following will be performed the 1-RM test to determine the load and intensity of individual training. After obtaining diagnostic data, active static stretching (SS) and specific warm-up (SW) will be applied on different days, followed by the benchmarking performance "Fran", through the assessment of heart rate, systolic blood pressure (SBP) and diastolic (DBP) and Borg scale. The results characterized a sampling with anthropometric and body composition parameters homogeneous. Specific warming showed a lower response of heart rate $(168.65 \pm 16.9 \mathrm{bpm})$ and subjective perception of exertion $(6.65 \pm 1.26)$ compared to static stretching (178.8 \pm 13.50 and $775 \pm 1.11$, respectively), however the pressure responses were not statistically different (SBP: $151.5 \pm 27.39 \mathrm{mmHg}$ vs. $154.5 \pm 10.99 \mathrm{mmHg}$; DBP: $116 \pm$ $5.98 \mathrm{mmHg}$ vs. $117.5 \pm 10.69 \mathrm{mmHg}$. In conclusion, specific warm-up promoted less cardiovascular work after benchmarking compared to static stretching.
\end{abstract}

Keywords: High intensity interval training; Warm up exercise; Muscle stretching exercises. 


\section{Resumen}

El calentamiento es una estrategia preparatoria importante para un buen rendimiento físico durante el entrenamiento, sin embargo, su aplicación metodológica en CrossFit aún es poco discutida. Este estudio tuvo como objetivo verificar el efecto agudo de diferentes estrategias de calentamiento sobre el rendimiento del punto de referencia de Fran en practicantes de CrossFit. Se trata de un estudio transversal con un enfoque cuantitativo, que consiste en un muestreo intencional no probabilístico de practicantes de CrossFit. Inicialmente, se evaluó el peso, la altura, el índice de masa corporal y el porcentaje de grasa para la caracterización de la muestra. En secuencia, se realizó la prueba de 1-RM para determinar la carga y la intensidad del entrenamiento individual. Tras la obtención de los datos diagnósticos, se aplicaron estiramientos estáticos activos (AL) y calentamiento específico (AQ), en diferentes días, seguido de la evaluación del rendimiento del benchmark "Fran", mediante la evaluación de la frecuencia cardíaca (FC), presión arterial sistólica (PAS) y diastólica (PAD) y escala de Borg. Los resultados caracterizaron un muestreo con parámetros antropométricos y composición corporal homogénea. El calentamiento específico mostró una respuesta de frecuencia cardíaca más baja $(168,65 \pm 16,9 \mathrm{lpm})$ y una percepción subjetiva de esfuerzo $(6,65 \pm 1,26)$ en comparación con el estiramiento estático (178,8 $\pm 13,50$ y $7,75 \pm 1,11$, respectivamente), sin embargo, las respuestas de presión fueron no estadísticamente diferentes entre sí (PAS: 151,5 $\pm 27,39 \mathrm{mmHg}$ frente a $154,5 \pm 10,99 \mathrm{mmHg}$; PAD: $116 \pm 5,98 \mathrm{mmHg}$ frente a 117,5 $\pm 10,69 \mathrm{mmHg}$ En conclusión, el calentamiento específico promovió menos trabajo cardiovascular después de la ejecución de referencia en comparación con el estiramiento estático.

Palabras clave: Entrenamiento por intervalos de alta intensidad; Ejercicio de calentamiento; Ejercicios de estiramiento muscular.

\section{Introdução}

O CrossFit pode ser definido enquanto método, como a junção de exercícios de diferentes modalidades esportivas, em uma variação constante, com a máxima funcionalidade possível e alta intensidade (Menezes, 2013). A modalidade CrossFit tem como pilar alcançar de maneira ampla e geral o condicionamento físico e motor a partir da máxima utilização das três principais vias metabólicas, a fosfagênica, a glicolitica e a oxidativa, a fim de preparar o indivíduo para qualquer trabalho físico conhecido ou desconhecido (Reis, Leão, Carvalho, \& Landim, 2020; Paine, Uptgraft \& Wylie, 2010).

Segundo Gerhart (2014), a metodologia CrossFit trabalha em torno das 10 capacidades físicas conhecidas, sendo elas, resistência cardiorrespiratória, resistência muscular localizada, força, flexibilidade, potência, velocidade, coordenação, agilidade, equilíbrio e precisão. Todavia, é percebido que o condicionamento dessas capacidades traz benefícios fisiológicos, melhorias no desempenho motor, além da redução de doenças cardiovasculares e diminuição da porcentagem de gordura.

Desta forma, o método CrossFit visa condicionar diferentes variáveis físicas durante o treinamento, tendo seu ápice referente ao condicionamento metabólico no momento do Wod (treino do dia). Nesta perspectiva, e inserido nos wods estão os benchmarks, que são treinos oficiais do CrossFit e servem de parâmetro avaliativo da evolução dos praticantes (Glassman, 2021).

Dentre os benchmarks originais, e mais comuns estão as "Girls" que recebem nomes de mulheres, e os "Hero Wods", feitos para homenagear soldados americanos mortos em ação. Um dos benchmarks mais tradicionais é a "Fran", caracterizada por trabalhar a potência envolvendo movimentos de levantamento de peso olímpico e ginástica em uma tarefa rápida.

Mcardle, Katch e Katch (2016), apontam que a primeira fase de uma atividade física é o aquecimento. Nessa perspectiva, Weineck (2003) diz que o aquecimento tem como propósito o aumento temperatura muscular, promovendo uma maior elasticidade no tecido, um aumento no débito cardíaco, melhorias na função do sistema nervoso central e recrutamento das unidades motoras neuromusculares.

Entre os diversos métodos de aquecimento descritos na literatura podem ser citados: o aquecimento ativo, que consiste na realização de movimentos de baixa intensidade mobilizando um fluxo sanguíneo considerável para os órgãos e sistemas orgânicos facilitando as ações musculares; o aquecimento passivo, classifica-se por não necessitar da execução de movimentos geradores de aumento na temperatura corporal, ele se dá através de aplicações externos de calor, tais como, banhos quentes, saunas e etc; o aquecimento geral, que resulta na mobilização de grandes grupamentos musculares associado com o aumento da temperatura corporal através da ativação do metabolismo em geral, na maioria das vezes o aumento da temperatura é obtido 
mediante a execução de exercícios cíclicos em ritmo calmo; e o aquecimento especifico, que está diretamente relacionado com a execução de ações neuromusculares específicas, sendo aplicadas posteriormente na atividade física proposta, ou seja, está diretamente envolvido com a musculatura recrutada na ação motora (Vieira \& Rosa, 2014).

Vários são os métodos de alongamento e diferenciam-se normalmente pela técnica de execução utilizada. Dentre as metodologias mais utilizadas como estratégia de aquecimento, em exercícios de força e resistência, está o método estático que além de parecer o mais utilizado devido sua facilidade de aplicação e aprendizado, oferece menores riscos de lesão. Podendo ser ativo, quando é estabelecido pela atividade muscular do próprio indivíduo envolvido na ação, sem ajuda externa; ou passivo, quando não ocorre contribuição ou contração ativa do sujeito submetido à ação, ou seja, o alongamento é totalmente promovido por forças externas, através do auxílio de um parceiro ou de um equipamento mecânico (Gomes, 2009).

Neste sentido, é observado que tanto o alongamento muscular estático ativo, quanto o aquecimento específico, são as duas principais e mais utilizadas estratégias preparatórias para a maioria das rotinas de treinamentos conhecidas (Simão, Giacomini, Dornelles, Marramom \& Viveiros, 2003).

A utilização do alongamento estático ativo tem sido alvo de discussões no que tange a sua utilização como estratégia inicial no treino de potência e força máxima. Alguns autores afirmam que sua aplicação interfere negativamente na curva tensão-comprimento do sarcômero e consequentemente no desempenho da força muscular durante o treino (Vieira \& Rosa, 2014; Enoka, 2000; Endlich et al., 2009) outros, pontuam que a prática do alongamento é essencial enquanto estratégia preparatória e insuficiente para influenciar no desempenho da força muscular (Simão et al., 2003; Silveira, Farias, Alvarez, Bif \& Vieira, 2011; Bastos et al., 2014).

No aquecimento específico são utilizados movimentos nos quais serão posteriormente executados na sessão de treinamento, com o objetivo de preparar as unidades motoras especificas, aumentar a capacidade coordenativa, como também um aumento da irrigação dos tecidos promovendo um aporte maior de oxigênio (Fermino et al., 2008).

Por ser uma modalidade esportiva relativamente nova, ainda são poucos os estudos que versam sobre suas aplicabilidades pedagógicas, e nada tem sido discutido quanto a influência destas estratégias preparatórias no treinamento de CrossFit.

Diante disso, este estudo teve como objetivo avaliar o efeito agudo de diferentes estratégias de aquecimento sobre o desempenho do benchmark Fran em praticantes de CrossFit.

\section{Metodologia}

Este estudo configura-se com delineamento transversal, de campo, com abordagem quantitativa e amostragem nãoprobabilística intencional (Pereira, Shitsuka, Parreira \& Shitsuka, 2018), realizada no Box CrossFit Mufasa, em Fortaleza, Ceará no período de maio a junho de 2019.

A amostra foi composta por 20 praticantes de Crossfit, com idade média de $28,95( \pm 4,97)$, sendo destes quatorze homens e seis mulheres. Foram considerados como critérios de inclusão a prática continua na modalidade por um tempo mínimo de seis meses, idade acima de 18 anos e experiência prática nos exercícios utilizados neste estudo. Esse critério foi adotado a fim de evitar o acometimento de dor muscular tardia, bem como falhas na determinação da carga de trabalho, devido à falta de coordenação necessária para a execução dos exercícios.

Foram excluídos desta amostra os praticantes com limitações físicas impedidos de realizar os testes propostos neste estudo, desvios posturais como hiperescoliose, hipercifose, hiperlordose e/ou joelhos valgos e varos, histórico recente de lesões articulares, ósseas ou musculares nos membros superiores e/ou inferiores ou que se recusassem em participar da pesquisa.

Os dados foram coletados em três etapas: 
Etapa 1: Dados de caracterização da amostra

Nesta etapa foram avaliados o peso, altura, o índice de massa corporal (IMC), o percentual de gordura (\%G), e o teste de 1-RM.

Etapa 2: Aplicação do alongamento estático ativo e análise do desempenho

Avaliação do desempenho do benchmark "Fran" após o alongamento estático ativo.

Etapa 3: Aplicação do aquecimento específico e análise do desempenho

Avaliação do desempenho do benchmark "Fran” após o aquecimento específico.

As etapas investigativas foram realizadas sequencialmente em dias distintos por todos os sujeitos da amostra.

Na etapa 1, o cálculo do IMC, foi obtido pelo coeficiente da massa corporal (em $\mathrm{kg}$ ) dividido pelo quadrado da altura em metros $(\mathrm{kg} / \mathrm{m} 2)$. Os valores do peso foram medidos com os indivíduos descalços e com roupas leves, em uma balança portátil digital posicionada no chão reto com o display automático acionada pelo toque dos pés, sendo capaz de registrar até $120 \mathrm{~kg}$ com uma precisão de $0,1 \mathrm{~kg}$. A altura foi aferida com a utilização de uma fita métrica com precisão de 0,5 , onde o ponto zero estava a nível do solo aderida em uma parede lisa (Machado \& Abad, 2012). O percentual de gordura foi avaliado através de aparelho de bioimpedância (Omron HBF-514C) que realizará a medição através de impedância bioelétrica terapolar de mãos e pés. As medidas antropométricas e de composição corporal da amostra estão apresentadas em valores médios e desvio padrão na Tabela 1 .

Tabela 1: Caracterização da amostra.

\begin{tabular}{cccc}
\hline Variáveis & Média & DP & CV \\
\hline Peso $(\mathrm{kg})$ & 75,52 & 13,36 & 0,17 \\
Altura $(\mathrm{cm})$ & 1,67 & 0,09 & 0,05 \\
IMC $\left(\mathrm{kg} / \mathrm{cm}^{2}\right)$ & 26,59 & 2,71 & 0,10 \\
$\% \mathrm{G}$ & 19,2 & 7,72 & 0,40 \\
\hline
\end{tabular}

Desvio padrão (DP); Coeficiente de variação (CV); índice de massa corporal (IMC); percentual de gordura (\%G). Fonte: Autores.

O teste de 1RM foi utilizado para determinação da força muscular do movimento "thruster", que compreende o grupamento muscular dos membros inferiores e membros superiores, e 1-RM de barra fixa para membros superiores isolados. Antes de se iniciar o teste de 1RM, os alunos realizaram aquecimento de oito repetições a $50 \%$ da carga estimada para a primeira tentativa do teste, seguido de três repetições a 70\%, onde o movimento a ser executado seguiu a cadência e amplitude delimitada pelo avaliador. Após 5 minutos de intervalo, foi realizado o teste de 1RM, acrescentando-se, quando necessário, 0,4 a $5 \mathrm{~kg}$, totalizando três a cinco tentativas, registrando como carga máxima aquela levantada em um único movimento (Uchida, Charro, Bacurau, Navarro \& Pontes, 2006). Foi usado 40\% da carga total de 1RM para assegurar que todos os praticantes estivessem com a mesma intensidade de carga durante a execução do benchmark nas etapas 2 e 3 .

A etapa 2 foi iniciada pela avaliação dos parâmetros de desempenho e intensidade em repouso, composta pela avaliação da frequência cardíaca, pressão arterial e escala de Borg.

A frequência cardíaca (FC) foi mesurada por frequencímetro digital e a pressão arterial (PA) foi aferida pelo uso de esfigmomanômetro calibrado e estetoscópio, por meio da técnica auscultatória. Os participantes foram orientados a não ingerir bebida alcóolica ou que contivessem cafeína no dia anterior, ter uma noite de sono satisfatória e evitassem fontes de estresse e exercício físico vigoroso, a fim de não interferirem na execução do teste bem como nos dados coletados.

A Escala CR-10 (Category-Ratio Scale) de Borg (1982) modificada por Foster et. al. (2001), foi utilizada para avaliar 
a percepção subjetiva de esforço (PSE) e quantificação das sensações durante cada nível do teste. A escolha deste protocolo se deu por ser o preditor comumente utilizada para medir a PSE ou intensidade de esforço em adultos.

Em sequência, foi aplicado o alongamento estático e ativo (AL) composto por quatro exercícios, dois para membros superiores e dois para membros inferiores, sendo aplicados em 3 séries, com duração de 30 segundos e intervalo em mesmo tempo. A intensidade foi controlada entre $70 \%$ a $90 \%$ do ponto subjetivo de desconforto (Lopes, Soares, Santos, Aoki \& Marchetti, 2015). Após o alongamento estático e ativo, a amostra realizou a benchmark Fran, caracterizada por unir movimentos usados no levantamento de peso olímpico, no caso o Thruster (união de um agachamento frontal com um desenvolvimento frontal, finalizando com a extensão total do corpo) e ginástica, por sua vez, o movimento de pull up (passagem do queixo na barra fixa). A benchmark Fran consiste em uma sequência de 21 thrusteres e 21 pull ups, em seguida, 15 thrusteres e 15 pull ups, finalizando com 9 thrusteres e 9 pull ups, com cargas oficiais para ambos os sexos, sendo $43 \mathrm{~kg}$ para homens e $30 \mathrm{~kg}$ para mulheres, realizados no menor tempo possível.

Ao final do benchmark, todos os parâmetros de desempenho foram reavaliados.

Estes procedimentos investigativos também foram realizados na etapa 3, sendo dessemelhante apenas a estratégia preparatória. Na etapa 3, foi realizado uma sequência de aquecimento específico (AE) com carga leve, composto por duas séries de 15 repetições com 40\% de 1-RM do movimento thruster, e duas séries de cinco a dez repetições de barra fixa, com intervalo de 30 segundos entre elas. Após a realização dos protocolos de aquecimento, foi dado dois minutos para início do teste.

Os dados de caracterização da amostra foram analisados e descritos, expressos como média e desvio padrão da média. Os dados estatísticos foram tabulados em uma planilha em excel (windows office 2016) em seguida analisados no programa SPSS for Windows 10, expressos como média e desvio padrão da média. Os parâmetros de desempenho foram submetidos ao teste t-Student, com nível de significância estabelecido de p <0,05.

Este estudo seguiu dentro das normas que regulamentam a pesquisa em seres humanos, do Conselho Nacional de Saúde - Ministério da Saúde, Resolução N 466/2012 e foi aprovado pelo CEP/IFCE sob o parecer n 3.436.616.

\section{Resultados e Discussão}

A modalidade CrossFit, inclui uma variedade de movimentos funcionais, cíclicos e ginásticos em constante variação e alta intensidade, exigindo do organismo um maior dispêndio metabólico. Por representar uma atividade intervalada de alta intensidade, e com características de superação e competividade, os benchmarks foram criados a fim de avaliar a melhoria da performance, bem como, mudanças corporais e fisiológicas, benefícios esperados com a prática da modalidade (Tibana, Almeida \& Prestes, 2015).

Butcher, Neyedly, Horvey e Benko (2015) ressaltam que os benchmarks são projetados para refletir diversas dimensões da aptidão física, sendo geralmente realizados e repetidos dentro de ciclos de treinamento para monitoração do progresso dos praticantes.

Neste estudo foram utilizados a avaliação da frequência cardíaca (FC), da pressão arterial sistólica (PAS) e diastólica (PAD), da percepção subjetiva de esforço (PSE), como parâmetros de análise de desempenho cardíaco e intensidade frente aos protocolos de alongamento estático e ativo (AL) e aquecimento específico (AQ).

A FC em repouso antecedente ao AL apresentou média de 70,3( $\pm 8,62) \mathrm{bpm}$ e ao AQ, média de 70,5( $\pm 6,95) \mathrm{bpm}$. Em

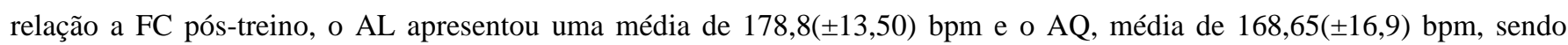
estatisticamente diferentes entre si (Figura 1). 
Figura 1: Avaliação da frequência cardíaca.

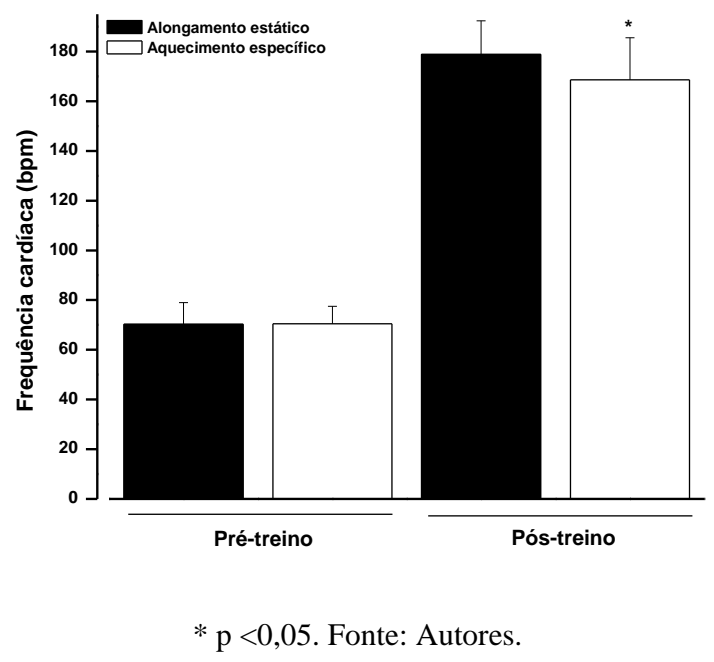

Os resultados mostraram um efeito cronotrópico cardíaco maior quando o AL é utilizado como estratégia preparatória em comparação ao AQ empregados neste estudo, sugerindo que o AQ pode reduzir o trabalho cronotrópico cardíaco ou que o AL amplia a participação desta variável em relação a execução deste benchmark.

A FC é regulada pelo centro cardiovascular, no bulbo, que recebe entradas (inputs) do córtex cerebral, do sistema límbico, dos proprioceptores, baroceptores e quimioceptores, além da participação química dos hormônios, de alguns íons, como o potássio, o cálcio e o sódio e da ação de outros fatores como idade, sexo, condicionamento físico e temperatura corporal (Mcardle, Katch \& Katch, 2016). Desta forma, as adaptações autonômicas gerenciadas pelo centro cardiovascular podem ter promovido a diferença cronotrópica visualizada entre os protocolos de aquecimento, como por exemplo, uma distinta participação do sistema límbico entre o $\mathrm{AL}$ e o AQ, ou uma maior participação dos proprioceptores durante o AQ. Uma outra possibilidade, seria uma ativação simpática progressiva promovida pela aplicação do AQ que pudesse gerar um efeito adaptativo cronotrópico no sistema cardiovascular.

$\mathrm{Na}$ análise da pressão arterial sistólica (PAS) antecedente ao benchmark, o AL obteve média de $113( \pm 9,78) \mathrm{mmHg}$ e o AQ média de $115( \pm 10) \mathrm{mmHg}$. A utilização do AL como estratégia preparatória ao benchmark apresentou uma média de PAS

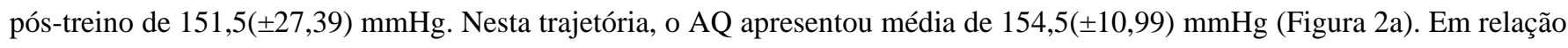
a pressão arterial diastólica (PAD) antes do benchmark, o AL obteve média de 89,5( 46,65$)$ mmHg e o AQ média de 81( $( \pm 7,88)$ mmHg. Após a execução do benchmark foram obtidos a média de PAD de $116( \pm 5,98)$ mmHg ao grupo precedido do AL de $117,5( \pm 10,69) \mathrm{mmHg}$ ao grupo precedido do AQ (Figura $2 \mathrm{~b})$. 
Figura 2: Avaliação da pressão arterial.

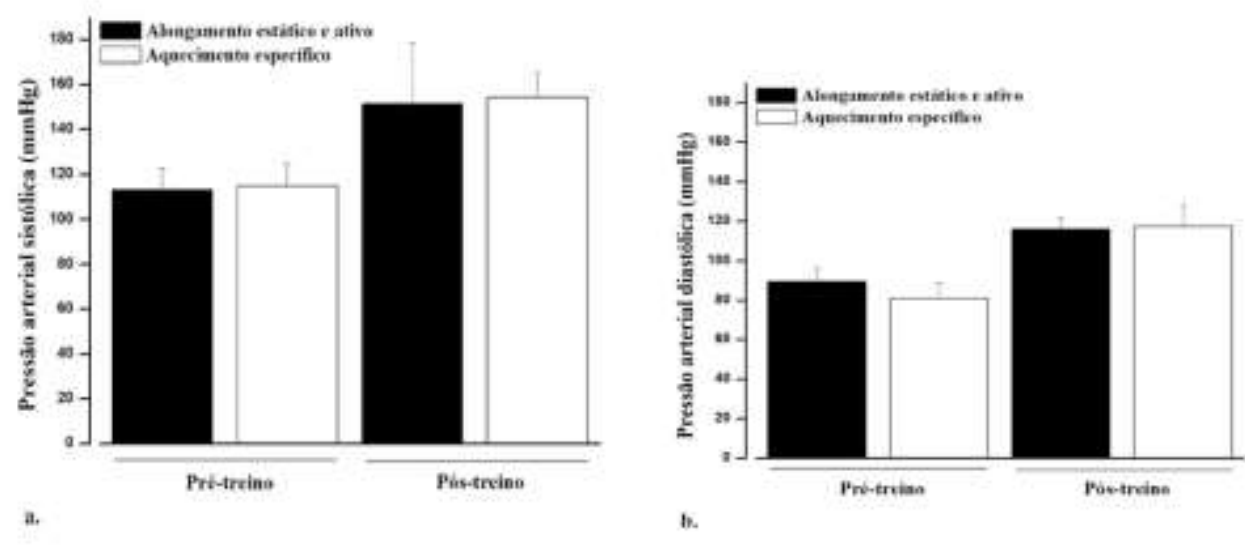

Fonte: Autores.

Em relação aos resultados pressórico-arteriais, tanto a pressão sistólica quanto diastólica, foram elevadas com a execução do benchmark, algo esperado pela natureza fisiológica diante à prática de exercícios físicos, todavia não apresentaram diferença estatisticamente significativa $(\mathrm{p}<0,05)$ quando comparadas entre si, caracterizando que os protocolos de aquecimento (AL e AQ) foram indiferentes às respostas pressóricas da amostra.

Os incrementos da PA pós exercícios, são determinados pelo aumento da resistência periférica sistêmica e pela interação do débito cardíaco. A PA é representada pela PAS, a mais alta pressão nas artérias estando associada com a sístole ventricular e consequentemente com o trabalho cardíaco, e pela PAD, menor pressão nas artérias ocasionada pela diástole ventricular, além de representar a resistência vascular periférica. A variabilidade da PA tende a ocorrer a partir de interações de diversos fatores, sendo na maioria influenciados pelo sistema nervoso autônomo (Mcardle, Katch \& Katch, 2016).

Exercícios realizados em alta intensidade, característica presente neste estudo e de grande apreço no CrossFit, tendem a apresentar componentes metabólitos que aumentam a resistência vascular periférica, consequentemente, ampliando a FC e sobretudo a PAS durante o exercício, além de fatores como a massa muscular recrutada, o padrão respiratório do praticante e o número de repetições realizadas (D'Assunção, Daltro, Simão, Polito \& Monteiro, 2007).

Vale ressaltar que a técnica de mensuração da PA utilizada neste estudo (auscultatório), embora seja bastante aceita e utilizada pela comunidade científica, pode apresentar fragilidade na caracterização da resposta pressórica em exercícios de alta intensidade, podendo ter sido subestimada durante o tempo de coleta.

A análise da PSE no AL apresentou uma média de 7,75( $\pm 1,11)$ e no AQ média de 6,65( $\pm 1,26)$, sendo estatisticamente diferente entre si (Figura 3).

A avaliação da PSE mostrou resultado semelhante ao obtido na análise da FC, caracterizando uma redução da percepção de esforço e sugerindo uma menor intensidade fisiológica quando o benchmark se utiliza do AQ como uma estratégia preparatória de treino.

A PSE tem sido amplamente utilizada como uma ferramenta de monitoramento da intensidade do treino por seu baixo custo e confiabilidade, sendo comumente associada a FC, entretanto, a relação entre valores atingidos na FC com os reportados na PSE ainda não é consensual (Nakamura, Moreira \& Aoki, 2010; Aissa, de Andrade Perez, Baldissera \& de Souza Lino, 2018). 
Figura 3: Avaliação da percepção subjetiva de esforço.

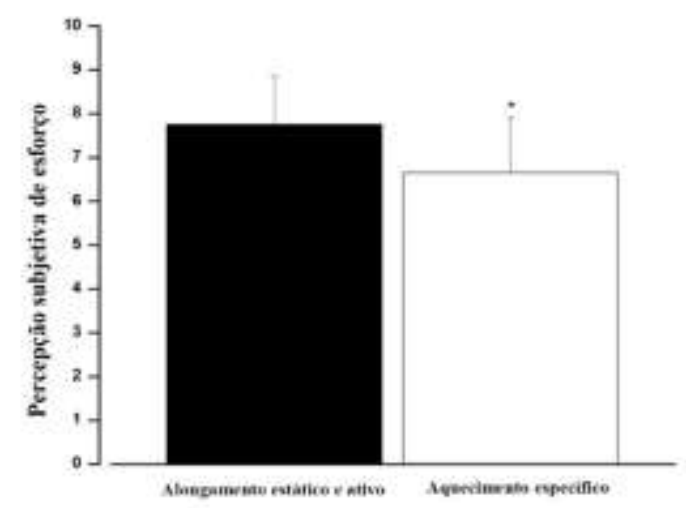

Legenda: $* \mathrm{p}<0,05$. Fonte: Autores.

Observa-se na literatura científica, um número crescente de pesquisas se utilizando o AL e o AQ no intuito de identificar qual a melhor estratégia de aquecimento a ser utilizada previamente em competições, atividades físicas ou testes de desempenho. Todavia, a maioria destes estudos se restringem as relações entre as variáveis de força e potência e pouco se tem discutido quanto as relações fisiológicas.

Estudos como os de Cramer et al. (2004) e Marek et al. (2005) afirmam que o alongamento enquanto estratégia de aquecimento, reduz o desempenho da força e da potência. Nesta trajetória, Bradley, Olsen \& Portas (2007) afirmam que o método de alongamento estático, utilizado em nosso estudo, reduz a força explosiva. Entretanto, os estudos de Simão et al. (2003), Silveira et al. (2011), Bastos et al. (2014), pontuam que a prática do alongamento é essencial enquanto estratégia preparatória e insuficiente para influenciar no desempenho da força muscular.

Fermino et al. (2008) e Ribeiro et al. (2007) avaliaram o AQ e compararam com o método de alongamento passivo, concluindo que ambos os métodos não exerceram influência no desempenho de uma sessão de exercícios resistidos. Abbud, Tabet e Dias (2013), afirmaram que o AQ não influenciou o desempenho do teste de repetições máximas a 70\% de 1RM no exercício de supino reto.

Estes estudos caracterizam respostas semelhantes entre o a prática do alongamento e do aquecimento no desempenho da força, todavia, nada se tem discutido quando ao desempenho cardiovascular que possa subsidiar essa discussão.

Diante disso, emergem alguns novos questionamentos como: será que esta resposta cardiovascular é algo peculiar a modalidade? O desempenho cardiovascular no treino de força possui a mesma resposta hemodinâmica do CrossFit? Haveria alguma relação motivacional intrínseca na resposta fisiológica promovida pelo aquecimento?

É importante destacar, que o benchmark proposto possui características intensas, sobretudo, alguns indivíduos da amostra alegaram informalmente desconfortos pós término do teste, tais como, náuseas, vertigem e hiperventilação, variáveis potencialmente intervenientes no momento da aferição, todavia, esses efeitos se normalizavam minutos depois.

Entre as limitações deste estudo encontram-se a escassa produção científica em torno do CrossFit, os poucos estudos de análise do comportamento cardiovascular em indivíduos treinados, especialmente em exercícios de alta intensidade que pudessem fomentar a discussão deste estudo e, a coleta da resposta pressórica mais precisa, uma vez que as relações temporais podem interferir no desempenho pressórico pós-treino.

Este estudo promove inúmeras reflexões quanto ao trato pedagógico no início do treinamento de Crossfit e nos leva a sugerir como objetos de futura investigação a avaliação com outros métodos de alongamento; a avaliação do desempenho cardiovascular a longo prazo; a inserção de um grupo sem qualquer estratégia preparatória; a mensuração do desempenho cardiovascular durante a execução do benchmark; avaliar outras variáveis potencialmente intervenientes, como tempo de 
tensão estimado, intervalos de recuperação e os aspectos nutricionais.

\section{Conclusão}

A amostra utilizada neste estudo apresentou parâmetros antropométricos e de composição corporal homogênea.

Os parâmetros de desempenho cardiovascular, cronotrópicos e pressóricos, bem como a percepção subjetiva de esforço, foram alterados com a prática do benchmark independente do protocolo de aquecimento utilizado.

$\mathrm{O}$ aquecimento específico apresentou uma menor resposta da frequência cardíaca e da percepção subjetiva de esforço em relação ao alongamento estático após a execução do benchmark, entretanto as respostas pressóricas não foram estatisticamente diferentes entre si.

Esses resultados apontam que o aquecimento específico promoveu um menor trabalho cardiovascular após a execução do benchmark em comparação ao alongamento estático e ativo.

\section{Referências}

Abbud, N., Tabet, J., \& Dias, M. (2013). Efeito do aquecimento específico em um teste de repetições máximas no exercício de supino reto. Revista Eletrônica da Faculdade Metodista Granbery, 14, 1-16.

Aissa, J. C., de Andrade Perez, S. E., Baldissera, V., \& de Souza Lino, A. D. (2018). Relação entre frequência cardíaca e percepção subjetiva de esforço em indivíduos entre 10 e 15 anos na natação. Revista Brasileira de Prescrição e Fisiologia do Exercício, 12(76), 597-604.

Bastos, C. L. B., do Rosário, A. C. S., Portal, M. D. N. D., Neto, G. R., Silva, A. J., \& da Silva Novaes, J. (2014). Influência aguda do alongamento estático no comportamento da força muscular máxima. Motricidade, 10(2), 90-99.

Bradley, P. S., Olsen, P. D., \& Portas, M. D. (2007). The effect of static, ballistic, and proprioceptive neuromuscular facilitation stretching on vertical jump performance. The Journal of Strength \& Conditioning Research, 21(1), 223-226.

Butcher, S. J., Neyedly, T. J., Horvey, K. J., \& Benko, C. R. (2015). Do physiological measures predict selected CrossFit ${ }^{\circledR}$ benchmark performance?. Open access journal of sports medicine, $6,241$.

Cramer, J. T., Housh, T. J., Johnson, G. O., Miller, J. M., Coburn, J. W., \& Beck, T. W. (2004). Acute effects of static stretching on peak torque in women. The Journal of Strength \& Conditioning Research, 18(2), 236-241.

D'Assunção, W., Daltro, M., Simão, R., Polito, M., \& Monteiro, W. (2007). Respostas cardiovasculares agudas no treinamento de força conduzido em exercícios para grandes e pequenos grupamentos musculares. Revista Brasileira de Medicina do Esporte, 13, 118-122.

Endlich, P. W., Farina, G. R., Dambroz, C., Gonçalves, W. L. S., Moysés, M. R., Mill, J. G., \& Abreu, G. R. D. (2009). Efeitos agudos do alongamento estático no desempenho da força dinâmica em homens jovens. Revista Brasileira de Medicina do Esporte, 15, $200-203$.

Enoka, R. M. (2000). Bases neuromecânicas da cinesiologia. São Paulo: Manole.

Fermino, R. C., Winiarski, Z. H., Rosa, R. J. D., Lorenci, L. G., Buso, S., \& Simão, R. (2005). Influência do aquecimento específico e de alongamento no desempenho da força muscular em 10 repetições máximas. Revista Brasileira de Ciência e Movimento, 13(4), 25-32.

Foster, C., Florhaug, J. A., Franklin, J., Gottschall, L., Hrovatin, L. A., Parker, S., \& Dodge, C. (2001). A new approach to monitoring exercise training. The Journal of Strength \& Conditioning Research, 15(1), 109-115.

Gerhart, H. D. (2013). A comparison of CrossFit training to traditional anaerobic resistance training in terms of selected fitness domains representative of overall athletic performance. Thesis (Master of Science). Indiana University of Pennsylvania, School of Graduate Studies and Research. Pennsylvania,

Glassman, G. (2021). O guia de treinamento CrossFit. <http://library.CrossFit.com/free/pdf/CFJ_L1_TG_Portuguese.pdf>

Gomes, A. C. (2009). Treinamento Desportivo: Estruturação e Periodização. (2a ed.), Artmed.

Lopes, C. R., Soares, E. G., Santos, A. L. R., Aoki, M. S., \& Marchetti, P. H. (2015). Efeitos do alongamento passivo no desempenho de séries múltiplas no treinamento de força. Revista Brasileira de Medicina do Esporte, 21, 224-229.

Machado, A. F. \& Abad, C. C. C. (2012). Manual de Avaliação Física. (2a ed.), Ícone.

Marek, S. M., Cramer, J. T., Fincher, A. L., Massey, L. L., Dangelmaier, S. M., Purkayastha, S., \& Culbertson, J. Y. (2005). Acute effects of static and proprioceptive neuromuscular facilitation stretching on muscle strength and power output. Journal of athletic training, $40(2), 94$.

Mcardle, W. D., Katch, F. I. \& Katch, V. L. (2016). Fisiologia do exercício: nutrição, energia e desempenho humano. (8a ed.), Guanabara Koogan.

Menezes, R. D. C. (2013). O forte do mercado: Uma análise do mercado de Fitness não convencional. Dissertação (Mestrado em Gestão Empresarial) - FGV

- Fundação Getúlio Vargas, Rio de Janeiro. 
Research, Society and Development, v. 10, n. 9, e30910918019, 2021

(CC BY 4.0) | ISSN 2525-3409 | DOI: http://dx.doi.org/10.33448/rsd-v10i9.18019

Nakamura, F. Y., Moreira, A., \& Aoki, M. S. (2010). Monitoramento da carga de treinamento: a percepção subjetiva do esforço da sessão é um método confiável. Journal of Physical Education, 21(1), 1-11.

Paine, J., Uptgraft, J., \& Wylie, R. (2010). CrossFit study. Command and General Staff College, 1-69. http://library.crossfit.com/free/pdf/CFJ _USArmy_Study.pdf.

Pereira, A. S., Shitsuka, D. M., Parreira, F. J. \& Shitsuka, R. (2018). Metodologia da pesquisa científica. UFSM. https://repositorio.ufsm.br/bitstream/ha ndle/1/15824/Lic_Computac ao_Metodologia-Pesquisa-Cientifica.pdf?sequence=1.

Reis, MGR, Leão, APN, Carvalho, LMF de, \& Landim, LA dos SR (2020). Perfil alimentar de praticantes de Crossfit: uma revisão da literatura. Research, Society and Development, 9 (11), e1439119579. https://doi.org/10.33448/rsd-v9i11.9579

Ribeiro, F. M., Oliveira, F., Jacinto, L., Santoro, T., Lemos, A., \& Simão, R. (2007). Influência aguda do alongamento passivo e do aquecimento específico na capacidade de desenvolver carga máxima no teste de 10RM. Fitness \& Performance Journal, 6(1), 5-9.

Simão, R., Giacomini, M. B., Dornelles, T. D. S., Marramom, M. G. F., \& Viveiros, L. E. (2003). Influência do aquecimento específico e da flexibilidade no teste de 1RM. Revista Brasileira de Fisiologia do Exercício, 2(2), 134-140.

Silveira, R. D. N. D., Farias, J. M. D., Alvarez, B. R., Bif, R., \& Vieira, J. (2011). Efeito agudo do alongamento estático em músculo agonista nos níveis de ativação e no desempenho da força de homens treinados. Revista Brasileira de Medicina do Esporte, 17, 26-30.

Tibana, R. A., Almeida, L. A., \& Prestes, J. (2015). Crossfit ${ }^{\circledR}$ risks or benefits? What do we know so far. Revista Brasileira de Ciência e Movimento, 23(1), $182-185$.

Uchida, M. C., Charro, M. A., Bacurau, R. F. P., Navarro, F. \& Pontes, F. L. (2006). Manual de musculação: uma abordagem teórico-prática do treinamento de força. 4. ed. São Paulo: Phorte.

Vieira, L. P., \& Rosa, C. G. S. (2014). Influência do aquecimento e alongamento na redução da força e potência muscular ao exercício resistido: Revisão bibliográfica. Amazônia: Science \& Health, 2(3), 38-43.

Weineck J. (2003). Treinamento Ideal. (9a ed.), Manole. 